\title{
Fatigue Analysis of Polycrystalline Materials Using Peridynamic Theory with a Novel Crack Tip Detection Algorithm
}

\author{
Ning Zhu ${ }^{1}$, Cemal Kochan ${ }^{2}$, Erkan Oterkus ${ }^{1, *}$ and Selda Oterkus ${ }^{1}$ \\ ${ }^{1}$ PeriDynamics Research Centre, Department of Naval Architecture, Ocean and \\ Marine Engineering, University of Strathclyde, Glasgow, UK \\ ${ }^{2}$ Mechanical Engineering Department, Dokuz Eylul University, Izmir, Turkey
}

\begin{abstract}
This study presents prediction of crack propagation in polycrystalline materials under cyclic loads using Peridynamic (PD) theory. The PD fatigue model utilizes the strainload cycle $(\varepsilon-\mathrm{N})$ data and introduces the "remaining life" of each bond calculated from its cyclic strain, which changes over time. The model also captures the traditional Paris law, which is widely used for computing the fatigue crack growth. Moreover, a new crack tip detection algorithm is introduced to adaptively detect the crack tip which is necessary for the PD fatigue model. Finally, several numerical cases are considered to investigate the effect of the strength of grain boundaries and the size of grains on the fatigue behaviour of polycrystalline materials.
\end{abstract}

Keywords: Peridynamics; fatigue; polycrystal; crack tip detection

\section{Introduction}

Marine structures are subjected to harsh marine environment including cyclic loads and corrosion. Fatigue, which occurs under cyclic loading, is one of the dangerous phenomena causing catastrophic failures below the static endurance limit of marine structures. Prediction of fatigue in engineering structures is usually challenging. Moreover, metallic materials used in marine structures are polycrystalline materials and their micro-structure at the crystal level can have a significant influence on the fatigue behaviour. Engineers and researchers have developed several different methods to overcome these challenges. During the late $20^{\text {th }}$ century, experimental methods were mainly used by researchers to analyse fatigue crack propagation. Reece et al. (1989) studied crack propagation in alumina under both static and cyclic loading conditions. They used electron microscope to scan the fracture surface of static and cyclic cracks and compared differences between them. Moreover, optical microscope was used in some other experimental studies to monitor cyclic fatigue crack paths by Dauskart et al. $(1987,1990)$ and Von Euw et al. (1972). On the other hand, researchers put significant effort to analyse the experimental results by using several different methods including the stress intensity factor (SIF) proposed by Irwin (Irwin 1957) and Paris' Law (Paris 1961) which was introduced to establish the fatigue crack propagation rate (Dauskart et al. 1987, 1990, Von Euw et al. 1972, Hopper and Miller 1977, Zheng and Hirt 1983, Pearson 1975). Another method which is also widely used is the stress-life (S-N) method. For example, the S-N curve obtained from the bending fatigue test of a high carbon-chromium specimen was used to understand the fracture modes (Shiozawa et al. 2001). Marines et al. (2003) used the S-N curve approach to modify the ultrasonic 
fatigue test results of the bearing steel AISI-SAE 52100. Moreover, Donahue et al. (1972) used crack opening displacement (COD) method to calculate SIF in order to estimate the fatigue crack growth rate. Crack closure approach was used by Wolf (1970), Lindley and Richards (1974) and Newman (1981) to analyse the fatigue crack growth. Rice (1967) summarised the mechanics of near crack deformation and fatigue crack propagation.

The development of technology and computational methods also provides researchers more options to deal with fatigue analysis problems. One of the most common methods, Finite Element Method (FEM), was used by Dhont (1998) to solve the stress field around the crack tip region and then use the S-N curve to predict the fatigue life of the structure. Dong (2001) used both FEM and S-N curve to study the fatigue behaviour of welded joints. Similarly, Paris' Law was used by Newman (1976) after obtaining the stress data around cracking area using FEM. Moreover, the Boundary Element Method (BEM) was also used by Gerstle et al. (1987) to compute the SIF, which was then used to calculate the fatigue crack growth rate based on Paris' Law. Since there are limitations for the FEM to deal with the crack growth problem, Cohesive Element Method (CEM)/ Cohesive Zone Method (CZM) has been developed to overcome this limitation. For instance, after generating displacement field using CEM, both Paris' Law and COD method were considered by Nguyen et al. (2001) to compare the propagation rate of both long and short cracks. Roe and Sigmund (2003) and Yang et al. (2001) used CZM to predict fatigue crack growth. However, the former used strain energy release rate to compute crack growth rate and the latter used Paris' Law. Extended Finite Element Method (XFEM) has also been widely used in fracture mechanics and Sukumar et. al. (2003) utilised XFEM to study the three-dimensional fatigue crack propagation. In addition to the stress related methods, strain energy related methods can also be used for fatigue analysis. Sih and Barthelemy (1980) provided expressions on calculation of crack growth rate based on strain energy density and claimed that using strain energy density to predict mixed mode fatigue crack growth rate is more convenient than SIFs. A strain energy density model was recently developed by Huffman et al. (2017) to predict fatigue crack propagation of a pressure vessel made of mild steel. Moreover, since J-integral method can be used to compute strain energy release rate around crack tip region, Dowling and Begley (1976) used Jintegral to study the elastic-plastic fatigue cracking behaviour. Although the methods described above are mainly focusing on macroscopic level, they could also be used for fatigue analysis at meso-scale (crystal level). For instance, microscopy and X-ray can be used to visualise and monitor the fatigue crack pattern at crystal level (Zhai et al. 2011, Chevalier et. al. 1999, Liu et. al. 2011). Moreover, in addition to CZM (Bouvard et al. 2009) and FEM (Kirane and Ghosh 2008, Liu et. al. 2011, Proudhon et. al. 2016), Molecular Dynamics (MD) (Potirniche et. al. 2005, Tang et. al. 2010) can also be used to study fatigue crack growth for crystals.

In this study, an alternative numerical approach called Peridynamic (PD) theory is used to study the fatigue crack propagation in polycrystalline materials for the first time in the literature. PD has several advantages with respect to some other existing approaches. Fatigue has three phases including nucleation, fatigue crack growth and final rupture. S-N curve approach can only provide fatigue life. Paris' law can only represent fatigue 
crack growth phase. On the other hand, PD can represent all three phases of fatigue process. Therefore, it is a more comprehensive approach. For the fatigue model used in this study, switching from nucleation to crack growth phase is done by automatically updating the location of the crack tip during the simulation. Therefore, a novel crack tip detection algorithm is developed.

PD theory was first introduced by Silling (2000) and being used by researchers to solve various different problems (Ni et. al. 2019, Imachi et. al. 2020, Gu et. al. 2019, Chowdhury et. al. 2019, Liu et. al. 2020, Zhang et. al. 2019, Fang et. al. 2019, Huang et. al. 2019, Wang et. al. 2018, Diyaroglu et. al. 2019, Yang et. al., 2019, Alpay and Madenci 2013, Oterkus et. al. 2012, Basoglu et. al. 2019). The first PD fatigue model was developed by Oterkus et. al. (2010). Moreover, Silling and Askari (2014) introduced another model which can reproduce the well-known Paris' Law and was used by Zhang et. al. (2016) to predict and compare the crack patterns with experimental results. A different PD fatigue model was introduced by $\mathrm{Hu}$ and Madenci (2017), which is based on the calculation of critical strain energy release rate to predict the fatigue life of a composite lamina. Our study utilizes the PD fatigue model given in Silling and Askari (2014) with a novel crack tip detection.

\section{Peridynamic Theory}

The equation of motion of a material point in PD theory can be expressed as (Madenci and Oterkus 2014):

$$
\rho(\mathbf{x}) \ddot{\mathbf{u}}(\mathbf{x}, t)=\int_{H_{\mathbf{x}}} \mathbf{f}\left(\mathbf{u}\left(\mathbf{x}^{\prime}, t\right)-\mathbf{u}(\mathbf{x}, t), \mathbf{x}^{\prime}-\mathbf{x}\right) d V_{\mathbf{x}^{\prime}}+\mathbf{b}(\mathbf{x}, t)
$$

where $\rho(\mathbf{x})$ represents the density of the material and $\ddot{\mathbf{u}}(\mathbf{x}, t)$ and $\mathbf{u}(\mathbf{x}, t)$ denote the acceleration and displacement of the material point $\mathbf{x}$ at time $t$, respectively. $\mathbf{f}\left(\mathbf{u}\left(\mathbf{x}^{\prime}, t\right)-\mathbf{u}(\mathbf{x}, t), \mathbf{x}^{\prime}-\mathbf{x}\right)$ is the PD bond force between material points $\mathbf{x}$ and $\mathbf{x}^{\prime} . V_{\mathbf{x}^{\prime}}$ represents the volume of the material point $\mathbf{x}^{\prime}$ ' which is located within the Horizon, $H_{\mathbf{x}}$ of the material point $\mathbf{x}$. The term "horizon" is a fundamental parameter in PD theory, which represents a specific region for a material point $\mathbf{x}$ defining the range of interactions between the material point $\mathbf{x}$ and its family members $\mathbf{x}^{\prime}$ (as shown in Figure 1) (Silling and Askari 2005). The size of the PD horizon, $\delta$, depends on whether the PD model can represent the physical mechanisms correctly (Seleson and Parks 2011).

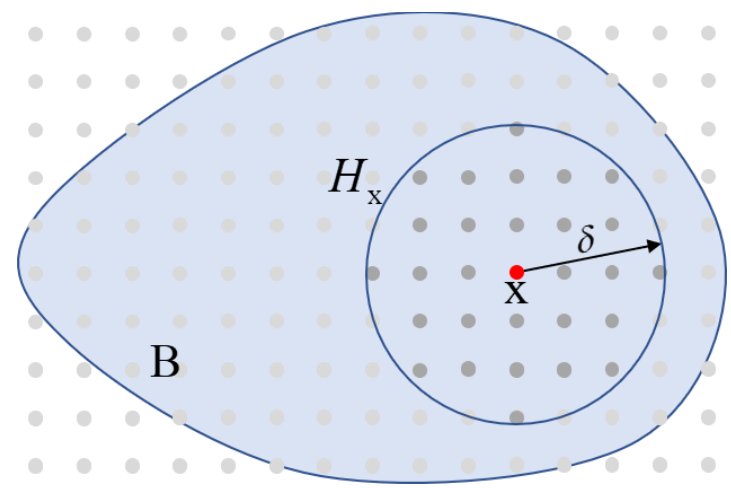


Fig. 1 Peridynamic Horizon

The expression of the PD bond force between material points $\mathbf{x}$ and $\mathbf{x}^{\prime}$ for an elastic material can be written as (Madenci and Oterkus 2014):

$$
\mathbf{f}\left(\mathbf{u}^{\prime}-\mathbf{u}, \mathbf{x}^{\prime}-\mathbf{x}\right)=c s \frac{\mathbf{y}^{\prime}-\mathbf{y}}{\left|\mathbf{y}^{\prime}-\mathbf{y}\right|}
$$

where $c$ represents the PD bond constant which includes material properties (Madenci and Oterkus 2014). $\mathbf{y}^{\prime}-\mathbf{y}$ term in Eq. (2) represents the relative position of the material point $\mathbf{x}^{\prime}$ with respect to the material point $\mathbf{x}$ after deformation. The term $s$ is the stretch of the bond between material points $\mathbf{x}^{\prime}$ and $\mathbf{x}$ which can be expressed as:

$$
s=\frac{\left|\mathbf{y}^{\prime}-\mathbf{y}\right|-\left|\mathbf{x}^{\prime}-\mathbf{x}\right|}{\left|\mathbf{x}^{\prime}-\mathbf{x}\right|}
$$

Fig. 2 shows the relationship between PD bond force $\mathbf{f}\left(\mathbf{u}^{\prime}-\mathbf{u}, \mathbf{x}^{\prime}-\mathbf{x}\right)$ and stretch $s\left(\mathbf{u}^{\prime}-\mathbf{u}, \mathbf{x}^{\prime}-\mathbf{x}\right)$ for a linear elastic brittle material. The $s_{0}$ in Fig. 2 denotes the critical stretch in PD theory, which means that a PD bond will be broken if $s>s_{0}$, i.e. bond force equals to zero. The damage of a material point can be calculated as:

$$
d=\frac{\text { number of broken bonds }}{\text { total number of bonds }}
$$

Surface correction is one of the important considerations in PD theory since material points close to the surfaces lack some family members which causes reduction in stiffness for these material points and should be corrected. The details of the surface correction procedure can be found in Madenci and Oterkus (2014).

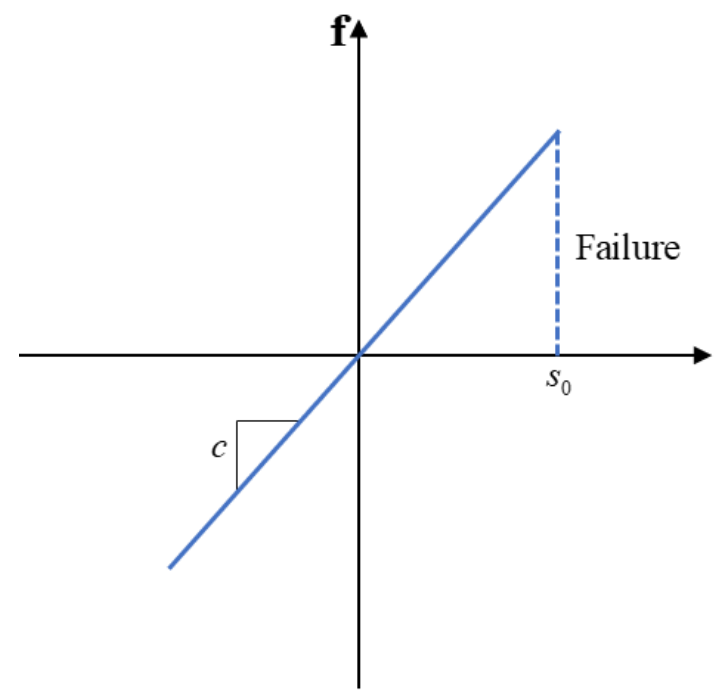

Fig. 2 Bond Constant and Critical Stretch for a Linear Elastic Brittle Material 


\section{Fatigue Model in Peridynamic Framework}

The PD fatigue model was recently introduced by Silling and Askari (2014) for linear isotropic materials which includes all three phases in fatigue failure; nucleation, fatigue crack growth and final rupture.

The failure algorithm described in previous section can only be used for the final rupture phase. For the nucleation and fatigue crack growth phases, a new failure algorithm called "remaining life" was introduced by Silling and Askari (2014). A new damage parameter $\lambda(\mathbf{x}, \xi, N)$ is utilised to monitor the remaining life of a bond $\xi$ which is associated with a material point $\mathbf{x}$, and its evolution with number of cycles $N$ can be expressed as (Silling and Askari 2014):

$$
\frac{d \lambda}{d N}(N)=-A \varepsilon^{m}
$$

where $A$ and $m$ are material constants which can be determined based on experimental data. $\varepsilon$ denotes the cyclic bond strain of the bond $\xi$, which can be computed as:

$$
\varepsilon=\left|s^{\max }-s^{\min }\right|
$$

where $s^{\max }$ and $s^{\min }$ are the stretch of the bond $\xi$ under the maximum and minimum loads in one cycle. It is assumed that the initial life of the bond $\xi$ at $N=0$ is 1 , i.e. $\lambda(0)=1$, and the bond breaks irreversibly when $\lambda(N) \leq 0$. Since the material is assumed to be linear elastic material, the cyclic bond strain can also be expressed by using only $s^{\max }$ and the load ratio $R=s^{\min } / s^{\max }$. Hence, Eq. (6) can be rewritten as:

$$
\varepsilon=\left|(1-R) s^{\max }\right|
$$

which means that only the maximum loading condition needs to be considered in simulations.

The initial phase of the PD fatigue model is Phase I: Nucleation. In this phase, the material constants $A$ and $m$ are calibrated with the experimental results (i.e. the S-N curve in strains) of the material as shown in Fig. 3. 


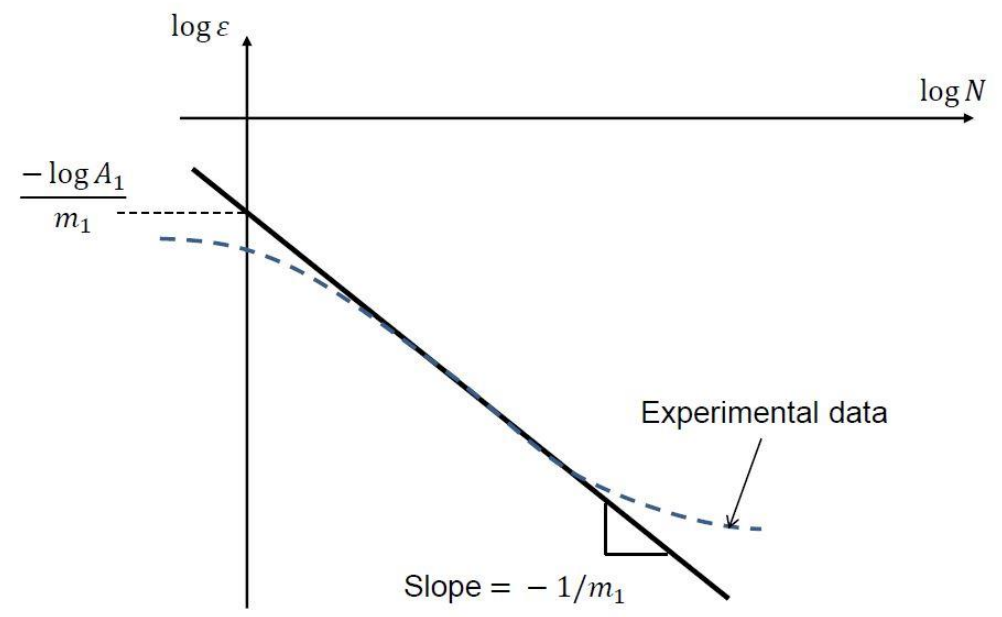

Fig. 3 Calibration of Material Constant for Phase I: Nucleation (Silling and Askari 2014).

Assuming that the first bond in the horizon $H_{\mathrm{x}}$ breaks at $N_{1}$ cycle, the corresponding cyclic strain range is $\varepsilon_{1}$. Since the initial remaining life of this bond is $\lambda_{1}(0)=1$, integrating Eq. (5) leads to:

$$
A_{1} \varepsilon_{1}^{m_{1}} N_{1}=1
$$

where $A_{1}$ and $m_{1}$ denotes the material constants for Phase I analysis. To obtain $A_{1}$ and $m_{1}$, a straight line is fitted to the experimental data in an S-N curve in terms of strains. Then, the constants can be obtained from the function of the fitted straight line. Hence, the first bond breaks when:

$$
N \geq \frac{1}{A_{1} \varepsilon_{1}^{m_{1}}}
$$

If there is a bond broken at $\varepsilon_{1}$ in Phase I, a new static analysis needs to be carried out for the same cycle. Since the strains of nearby bonds will increase, if the strain of a nearby bond is larger than $\varepsilon_{1}$, then that bond should also be broken and another static solution should be obtained until there are no more bonds broken.

The second phase of the PD fatigue model is Phase II: fatigue crack growth. Unlike Phase I, in which Eq. (5) is used to evaluate the fatigue life of a bond, the remaining life $\lambda_{i j}^{N}$ associated with the material points $\mathbf{x}_{i}$ and $\mathbf{x}_{j}$ for a particular cycle $N$ can be evaluated using backward finite difference approach. Therefore, Eq. (5) can be rewritten as (Zhang et. al. 2016):

$$
\lambda_{i j}^{N}=\lambda_{i j}^{N-1}-A_{2}\left(\varepsilon_{i j}^{n}\right)^{m_{2}}
$$

with 


$$
\lambda_{i j}^{0}=1
$$

where $\varepsilon_{i j}^{N}$ represents the cyclic strain of bond $\xi_{i j}$ at a particular cycle $N$, and $A_{2}$ and $m_{2}$ are material constants for Phase II analysis. In order to determine these two material constants, the PD remaining life evolution law needs to be calibrated with the wellknown Paris' Law (Silling and Askari 2014), whose data can be obtained from experiments. It is assumed that the evolution law for the remaining life is only suitable to use for the bonds within the horizon of material points that are in Phase II or cracktip. The details of how to obtain $A_{2}$ and $m_{2}$ constants can be found in Silling and Askari (2014) and Zhang et al. (2016).

The transformation of PD fatigue analysis from Phase I (Nucleation) to Phase II (Fatigue crack growth) happens if there is a material point within the horizon (including itself) with a damage value of $d \geq 0.5$. Note that $d \geq 0.5$ indicates existence of a crack in the structure. Therefore, if there is a pre-existing crack in the model, the material points located within the horizon of the crack tip will directly switch to Phase II since there are material points whose damage values already reach to 0.5 .

\section{Crack Tip Detection Algorithm}

As described in the previous section, the material points which are located within horizon of the crack tip will directly switch from Phase I to Phase II if a pre-existing crack exists in the model. Hence, it is essential to automatically update the location of the crack tip during the simulation. "bwmorph" function with "endpoints" option was used by Zhang et. al. (2016) after converting the PD damage map to black and white images by using the Matlab software. There are different crack tip detection (or tracking) algorithms developed in CZM and FEM (Saloustros et. al. 2015, Sancho et. al. 2007, Wang and Xu 2015, Cervera and Chiumenti 2006). However, there are very few crack tip detection algorithms available in PD theory since it is usually not necessary to determine the crack tip in PD theory. The crack tip detection algorithm developed in this study is similar to the one introduced by Cervera et. al. (2010). There are three main steps when using crack tip detection algorithm in PD framework. Firstly, a mesh is generated using PD bond to connect a material point with its neighbouring material points only. In other words, there are no bonds exceeding length of $\Delta x$ in this mesh. Therefore, the model is discretised into unit blocks which contain four material points and four bonds with a length of $\Delta x$ in each block. Secondly, the four bonds in the block are checked in every PD integration step to see if any of them is broken. If at least one bond is broken, the status of this block is marked as "damaged" (shown as " $T$ " in Figure 4). A "damaged" block means the crack tip has already either reached or passed this block. Thirdly, for each "damaged" block, its neighbouring blocks need to be checked to see how many of these blocks are "damaged". Hence, the crack tip is located at the damaged block which has only one damaged neighbouring block. 


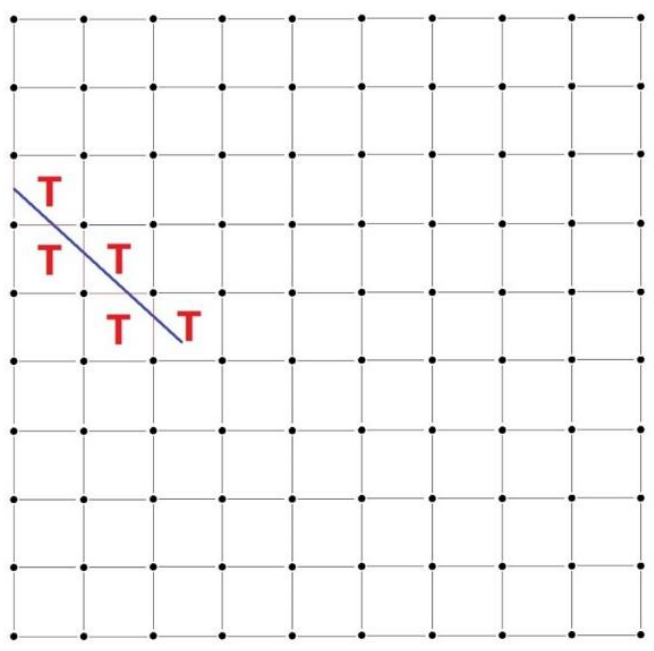

Fig. 4 PD mesh used in the crack tip detection algorithm. The blue line represents the pre-existing crack and " $T$ " means damaged block.

\section{Fatigue Analysis Procedure for a Polycrystalline Material}

The PD fatigue analysis procedure is summarised in the flowchart given in Fig. 8. 


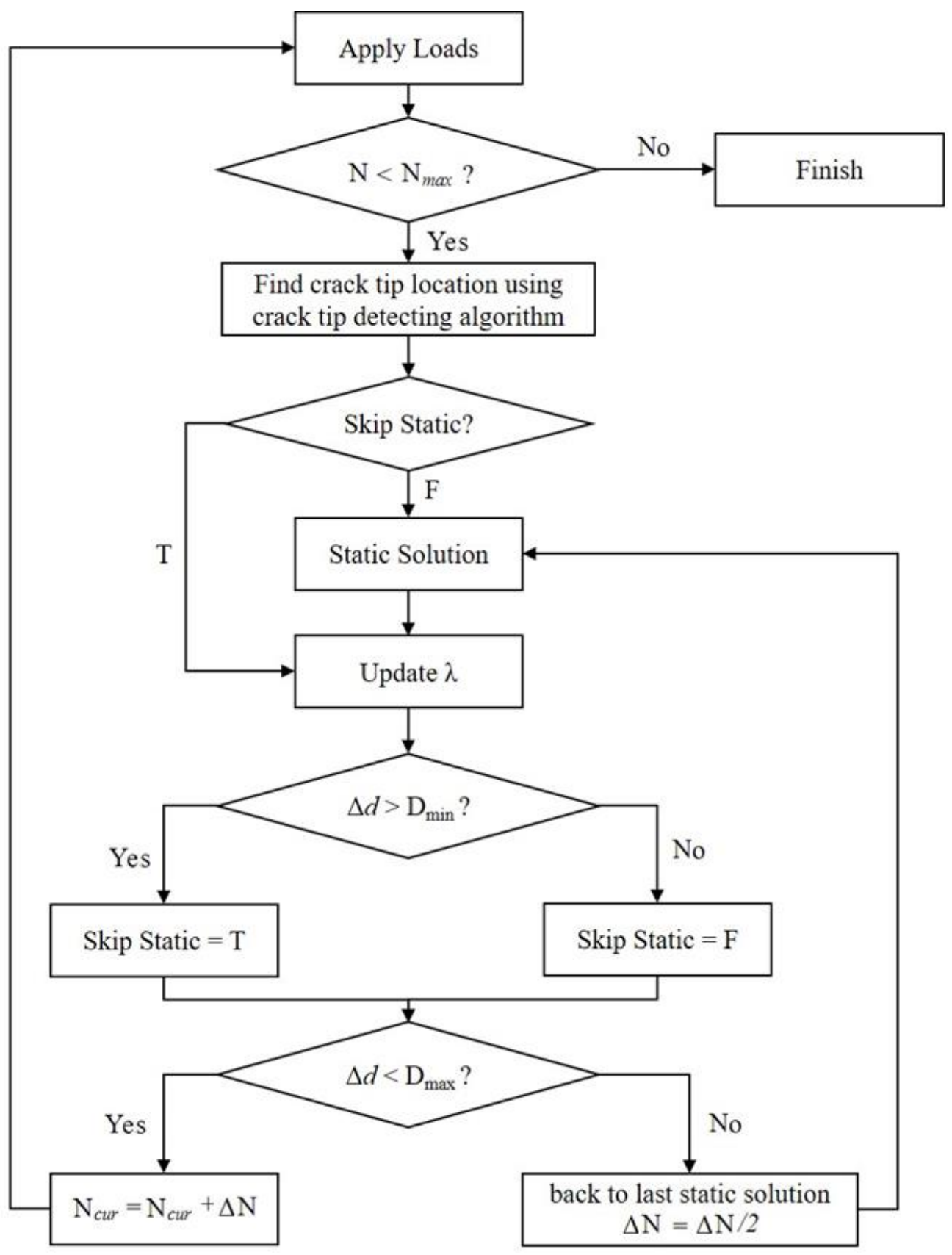

Fig. 8 Flowchart for PD fatigue analysis with critical damage factors.

In Fig. $8, D_{\min }$ and $D_{\max }$ represent the critical damage factors which were introduced by Zhang et. al. (2016). The purpose of introducing these two parameters is not only to determine the correct crack path with minimum number of static solutions, but also to prevent from breaking too many bonds within one static solution step which can make the solution unstable. In each static solution step, the two critical damage factors and the maximum damage difference $(\Delta d)$ of a material point, which is located within a certain area from the crack tip, are compared between the previous static solution step and the current one to determine the next action in the flowchart. The location of the crack tip is also updated for each static solution step in order to determine the material points that are transferred to the fatigue crack growth stage. 


\section{Numerical Results}

\subsection{Analysis of a Plate with a Central Crack Using Crack Tip Detection Algorithm}

Before starting to use PD fatigue model to analyse a polycrystalline material, the new crack tip detection algorithm is tested by simulating the same problem presented in Madenci and Oterkus (2014). A square plate is considered having dimensions of 0.05 $\mathrm{m}$ width and $0.0001 \mathrm{~m}$ thickness with a $0.01 \mathrm{~m}$ long pre-existing central crack. The plate is discretised with 500x500 material points. Moreover, three additional layers of material points are included at the top and bottom edges (as shown in Figure 5) in order to apply $20.0 \mathrm{~m} / \mathrm{s}$ velocity boundary condition to these edges. The horizon $\delta$ is set as 3.015 times the spacing between material points, and the critical stretch is $s_{0}=0.04472$. There are 1250 time steps in the simulation and the time step size is $\Delta t=1.3367 \times 10^{-8} \mathrm{~s}$.

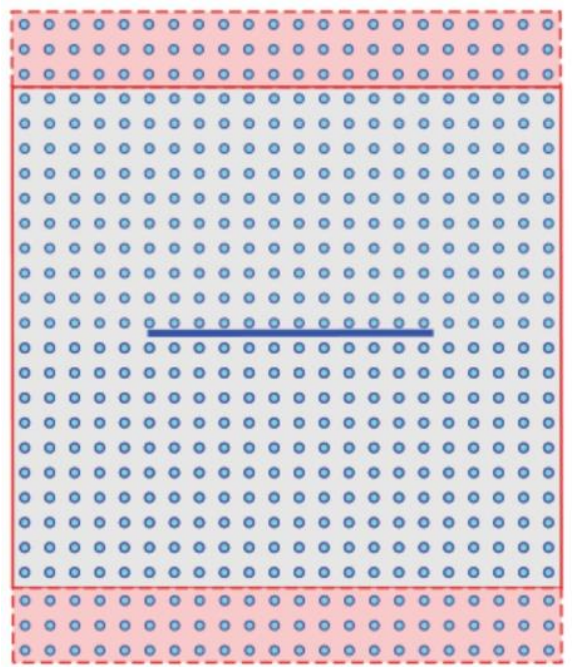

Fig. 5 Plate with a pre-existing central crack

In Madenci and Oterkus (2014), local damage value was used to determine the location of the crack tip, which was based on any material point's damage value exceeding $d=0.38$ along the $x$-axis. Note that this approach is limited to self-similar crack growth and the crack path should be known in advance. The crack growth with respect to time is shown in Fig. 6 and the crack growth speed can be obtained as $1650 \mathrm{~m} / \mathrm{s}$.

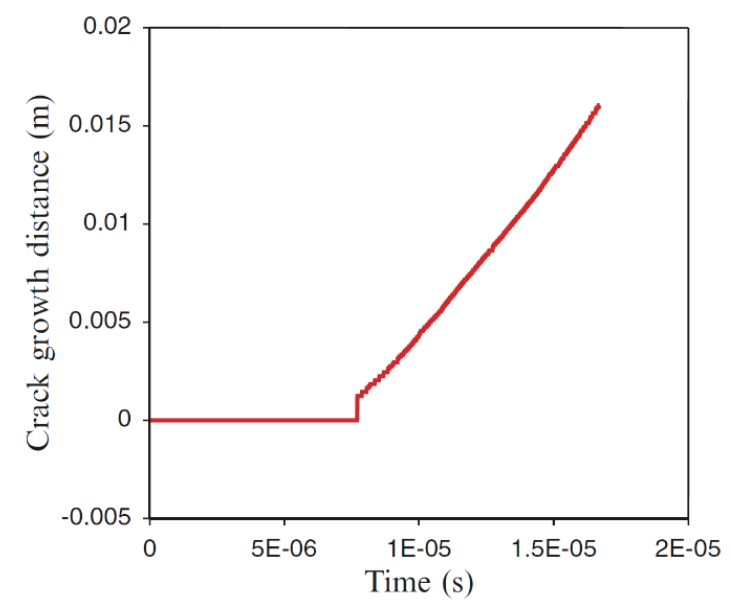

Fig. 6 Crack growth vs. Time 


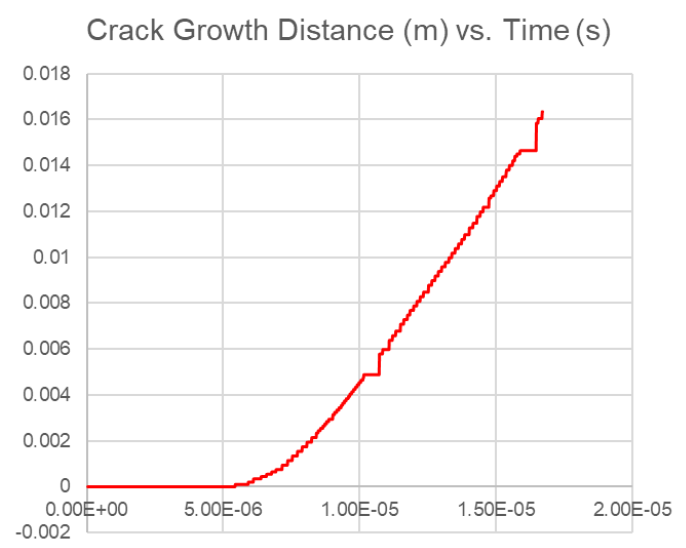

Fig. 7 Crack growth vs. time using the new crack tip detection algorithm

The crack growth data for the same problem can also be obtained by using the new crack tip detection algorithm as shown in Fig. 7. The crack growth speed can be calculated from the slope of the straight line as $1641 \mathrm{~m} / \mathrm{s}$. By comparing the results with those in Madenci and Oterkus (2014), it can be concluded that the results are in good agreement.

\subsection{PD Fatigue Analysis}

The geometry of the model used in PD fatigue analysis is given in Fig. 9. The material used in the analysis is the cold rolled SAE 1020 steel with Young's modulus of E = 205 GPa and Poisson's ratio of 0.29. According to Zhang et. al. (2016), since there is a preexisting crack in the model, the whole simulation will automatically switch to the crack growth phase. Hence, the key parameters used in the analysis are $A_{2}=9.53 \times 10^{6}$ and $m_{2}=2.1$. The horizon size is $\delta=1.2 \mathrm{~mm}$ and $\delta / \Delta x=4$. After studying the crack pattern with various of horizon sizes and grid sizes, and comparing the analysis results with experimental results, these horizon and discretisation size values are decided as they can produce reasonably accurate results with relatively less time. The applied force is $P=6000 N$. The bond constant is specified as $c=1.1329 \times 10^{24} \mathrm{~N} / \mathrm{m}^{6}$. The static solution is obtained by directly assigning the inertia term being zero and solving a matrix equation.

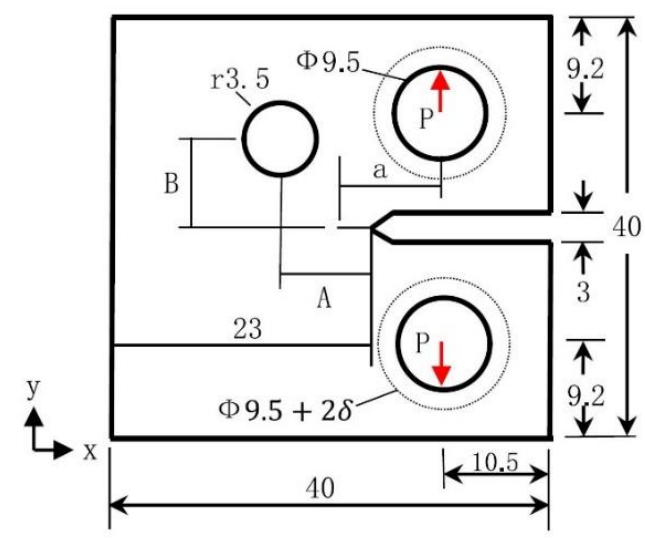

Fig. 9 Geometry of the model used in PD fatigue analysis (Unit: mm) (Zhang et. al. 2016). 
Three different polycrystalline models are considered in this study with 25, 100 and 400 grains (crystals) randomly distributed within the model, respectively. The polycrystalline models are generated using Voronoi diagram as shown in Fig. 10. To investigate various fracture modes of polycrystalline materials, the "interface strength coefficient ( $\beta$ )" was first introduced by Askari et. al. (2008) and then discussed by De Meo et. al. (2016) and Zhu et. al. (2016). Similarly, the interface strength coefficient is also considered in this study to investigate the effect of interface strength on the crack patterns. However, unlike $\beta$ coefficient described in Askari et. al. (2008), the $\beta$ coefficient used in this study is defined as:

$$
\beta=\frac{A_{2}^{G I}}{A_{2}^{G B}}
$$

where $A_{2}^{G B}$ and $A_{2}^{G I}$ denote the material constants used in the Phase II PD fatigue analysis for the bonds that cross the grain boundaries and the bonds that are located within the grains, respectively. Here, GB and GI represent the grain boundary and the grain interior, respectively. Therefore, when $\beta<1.0, A_{2}^{G B}$ is larger than $A_{2}^{G I}$, which means the remaining life of the cross-grain bonds will decrease faster than the in-grain bonds, assuming that the bonds have the same cyclic strain value. In other words, $\beta<1.0$ means the grain boundary is weaker than the grain interior. On the other hand, $\beta>1.0$ represents that the grain boundary is stronger. This study considers five different interface strength coefficient, $\beta$, values $(0.1,0.5,1.0,2.0,10.0)$ in addition to three different total numbers of grains $(25,100$ and 400$)$.

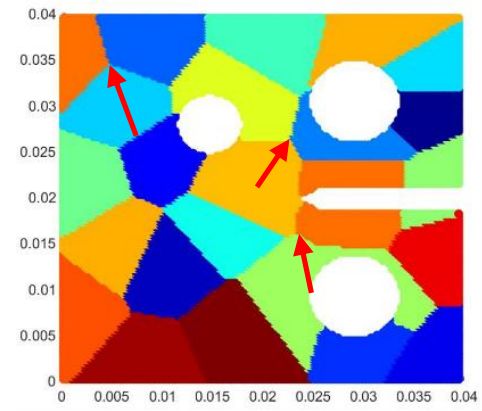

(a)

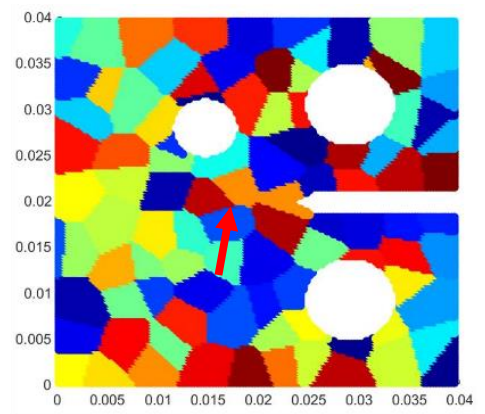

(b)

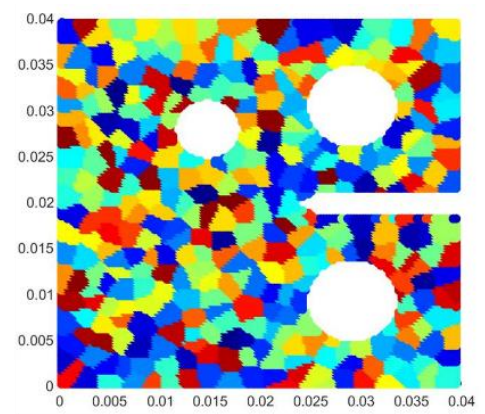

(c)

Fig. 10 Polycrystalline models for PD fatigue analysis. (a): 25 grains, (b): 100 grains and (c): 400 grains.

\subsection{Effect of interface strength coefficient on crack patterns}

The first analysis aims to investigate the crack patterns of intergranular and transgranular fracture modes by changing the interface strength coefficient value. 


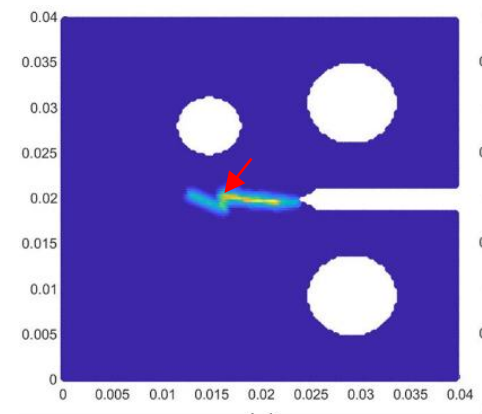

(a)

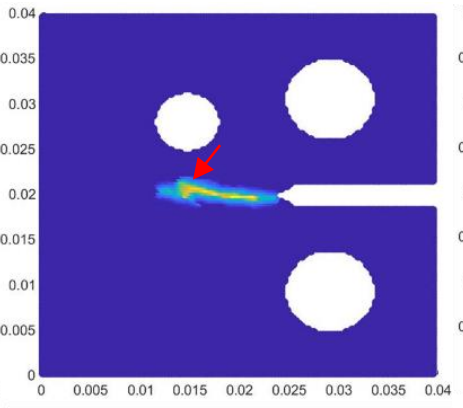

(b)

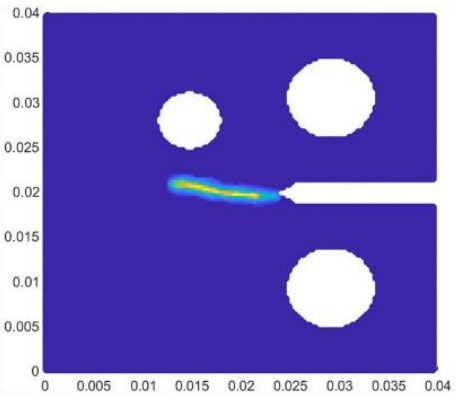

(c)

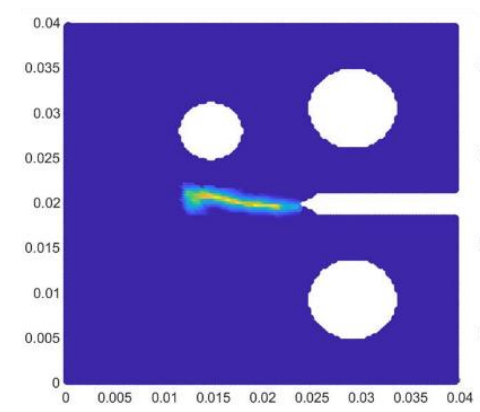

(d)

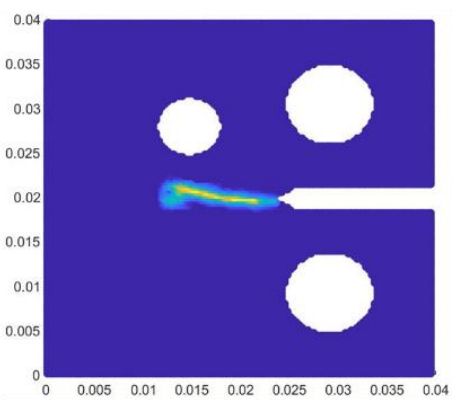

(e)

Fig. 11 Crack patterns of polycrystal with 25 grains. (a) $\beta=0.1, N=246 k$ cycles , (b)

$$
\begin{aligned}
& \beta=0.5, N=331 k \text { cycles, (c) } \beta=1.0, N=345 k \text { cycles, (d) } \\
& \beta=2.0, N=431 k \text { cycles, and (e) } \beta=10.0, N=429 k \text { cycles }
\end{aligned}
$$

By comparing the results in Figs. 11(a,b) with (c), it can be seen that there is a sharp turn of the crack pattern (red arrow) in both Figs. 11(a,b). This is because the crack firstly propagates within the grain until it reaches close to the grain boundary, and since the grain boundary in Figs. 11(a,b) is weaker, for the crack pattern it is much easier to follow the grain boundary (intergranular fracture mode) compared to Fig. 11(c).

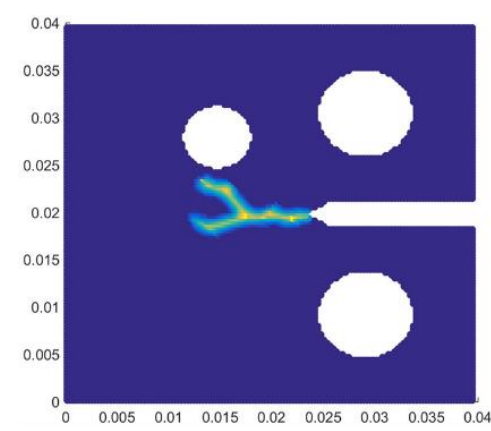

(a)

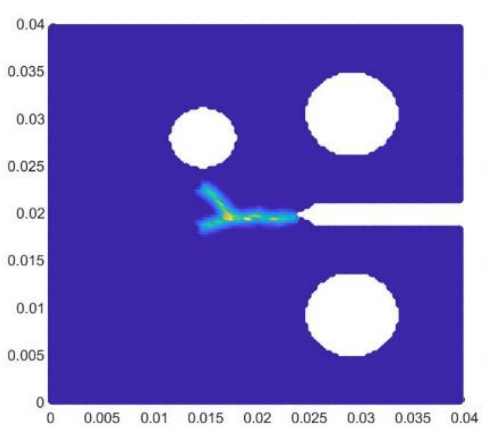

(b)

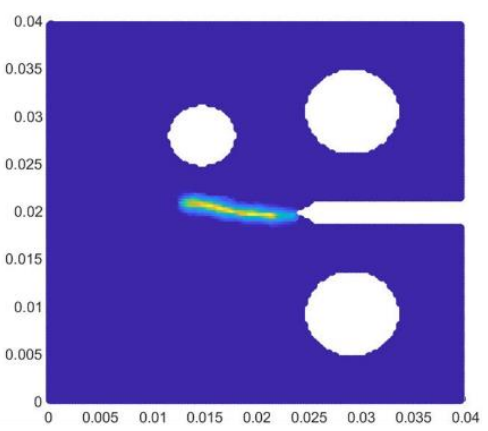

(c)

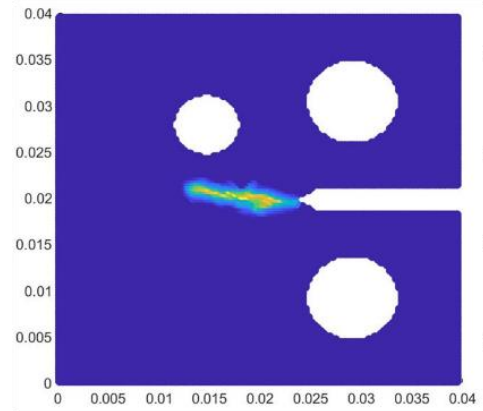

(d)

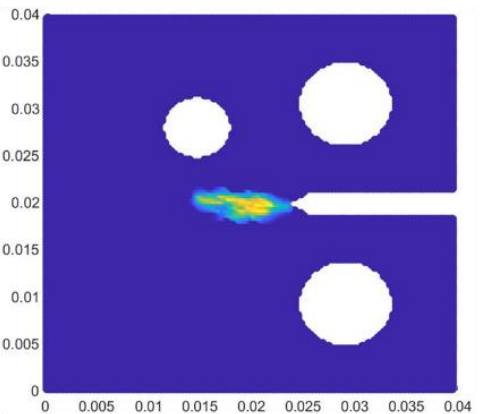

(e) 
Fig. 12 Crack pattern of polycrystal with 100 grains. (a) $\beta=0.1, N=48 k$ cycles, (b)

$$
\begin{aligned}
& \beta=0.5, N=174 k \text { cycles, (c) } \beta=1.0, N=345 k \text { cycles, (d) } \\
& \beta=2.0, N=453 k \text { cycles, and (e) } \beta=10.0, N=784 k \text { cycles }
\end{aligned}
$$

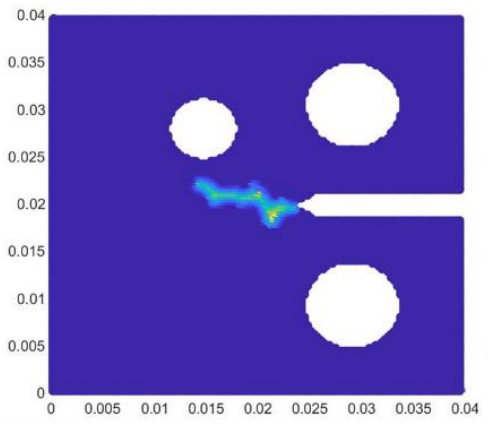

(a)

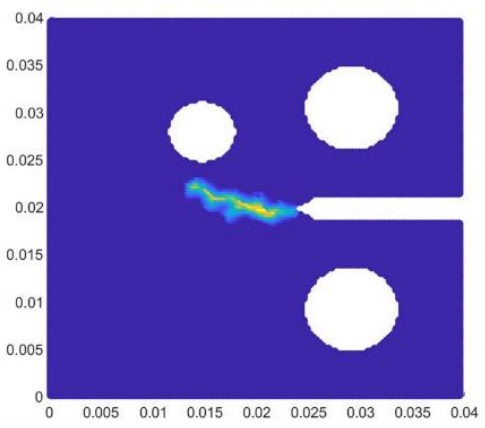

(b)

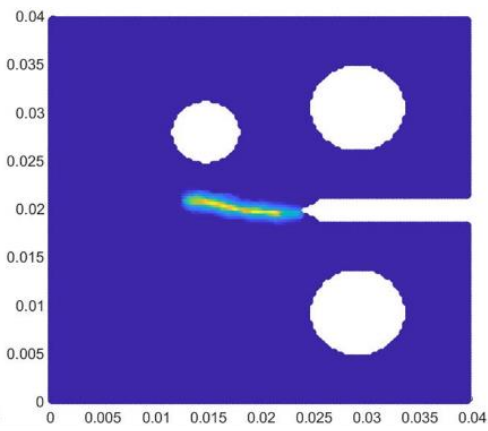

(c)

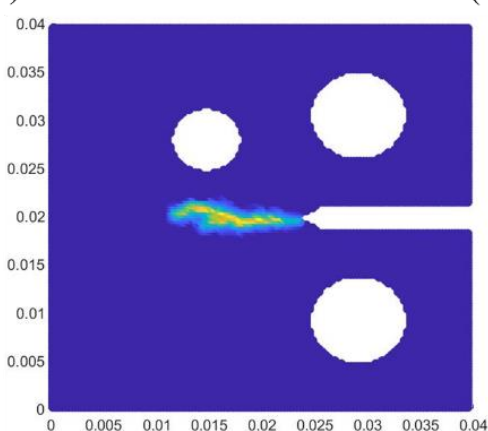

(d)

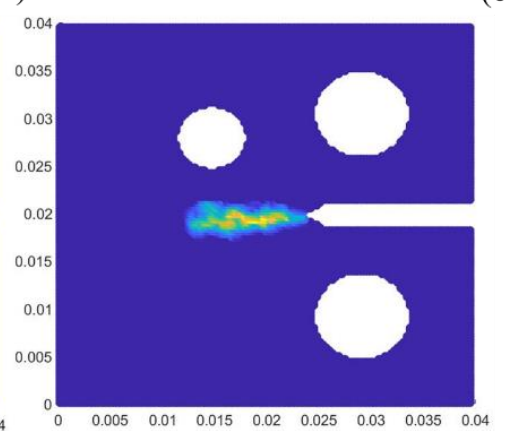

(e)

Fig. 13 Crack pattern of polycrystal with 400 grains. (a) $\beta=0.1, N=51 k$ cycles, (b) $\beta=0.5, N=214 k$ cycles, (c) $\beta=1.0, N=345 k$ cycles, (d) $\beta=2.0, N=512 k$ cycles, and (e) $\beta=10.0, N=846 k$ cycles

The influence of interface strength coefficient on the crack pattern can be seen more clearly in Figs. 12 and 13 then Fig. 11. When $\beta<1.0$ (Figure 12(a), 12(b), 13(a) and 13(b)), which means the bonds across the grain boundaries are much weaker than those located within the grain and the crack will more likely to propagate along grain boundaries compared to the crack patterns when $\beta>1.0$ (Figs. 12(d), 12(e), 13(d) and 13(e)). When $\beta=1.0$ (Figure 11(c), Figure 12(c) and Figure 13(c)) the grain boundary and grain interior properties are same and the crack propagates smoothly with respect to other cases. Note that microstructural characteristics are not important for this condition.

\subsection{Effect of crystal size on propagation speed}

The aim of the comparisons discussed in this section is to determine how the crystal size can affect the crack propagation speed. Total number of cycles when crack propagates to a similar location ( $x \cong 12.5 \mathrm{~mm}$ ) are compared between different number of grains. 


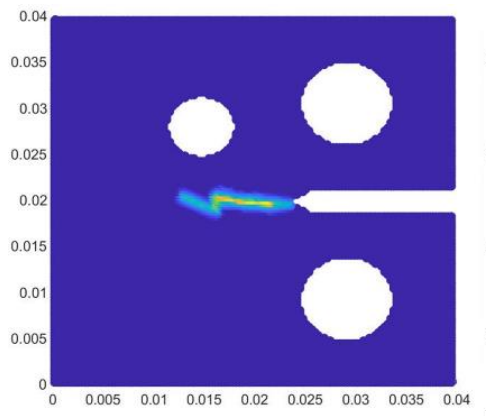

(a)

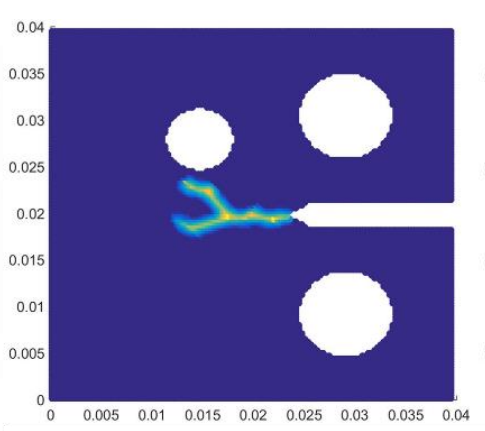

(b)

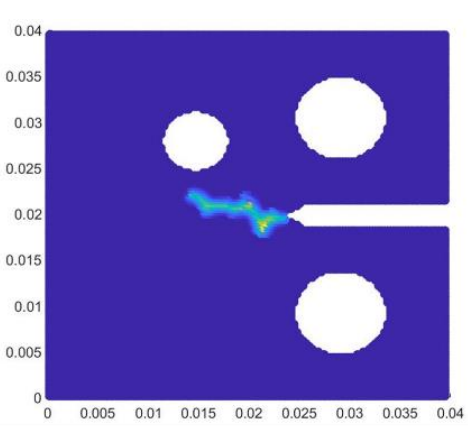

(c)

Fig. 14 Crack pattern of the polycrystal when $\beta=0.1$. (a) 25 grains, $N=246 k$ cycles , (b) 100 grains, $N=48 k$ cycles , and (c) 400 grains, $N=51 k$ cycles .

Figure 14 compares the crack patterns and total number of cycles with $\beta=0.1$ for 25 , 100 and 400 grains, respectively. It can be seen from the results that the 25 grain model requires more cycles for the crack to propagate to the same location with respect to other grain models (where $x \cong 12.5 \mathrm{~mm}$ ). As shown in Fig. 10(a), the pre-existing crack tip is located inside a grain. Since, the size of the crystal is much larger in 25 grain case, the crack needs to propagate much longer to reach the boundary. Hence, the crack tip in Fig. 14(a) requires significantly more cycles (246k cycles) to reach the target location than Fig. 14(b) ( $48 k$ cycles ). By increasing of the total number of crystals, the number of bonds which cross the grain boundaries increases. For the $\beta<1.0$ case, which means the grain boundaries are weaker, the model with more crystals have more weaker bonds than the model with less crystals, i.e. the crack is much easier to propagate. Hence, it is expected that the number of cycles required for the crack tip to reach the target location in the model with more crystals will be less than the model with less crystals. However, according to the results given in Figs. 14(b) and 14(c), the 400 crystal model requires more cycles than the 100 crystal model, which is the opposite to what it was expected. This is because with the increase in total number of crystals within the model, there will be more points that are located at the boundary of multiple grains (as shown in red arrows in Fig. 10(a)). When the crack tip reaches these key points, a certain number of cycles is required for the crack tip to "decide" which direction to propagate. With the increase in number of key points, the more cycles are required for the crack tip to "decide" the propagation direction. Therefore, the number of cycles required for the crack in 400 crystal model to reach the target location is larger than that the 100 crystal model. When the interface strength coefficient $\beta$ is changed from 0.1 to 0.5 , the results remain similar as shown in Fig. 15. 


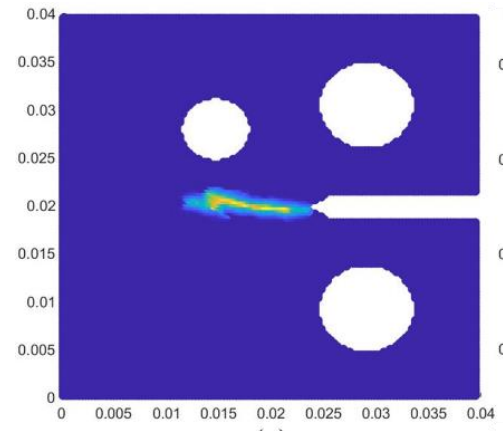

(a)

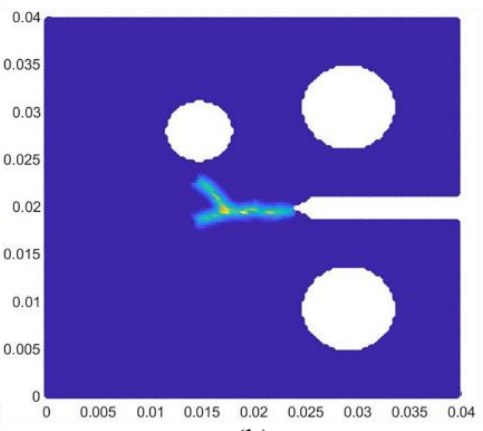

(b)

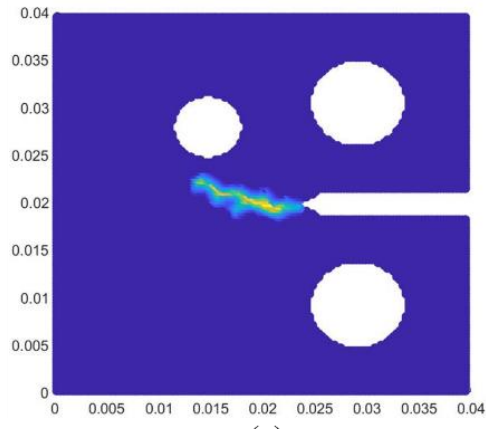

(c)

Fig. 15 Crack pattern of polycrystal when $\beta=0.5$. (a) 25 grains, $N=331 k$ cycles, (b) 100 grains, $N=174 k$ cycles, and (c) 400 grains, $N=214 k$ cycles .

For $\beta>1.0$ case, since the bonds that cross the grain boundaries become stronger than those are located within the grains, the crack will more likely to propagate through grains and trans-granular fracture occurs. Hence, with the increase in total number of crystals more bonds cross grain boundaries and more cycles are required to reach the target location as shown in Figs. 16 and 17.

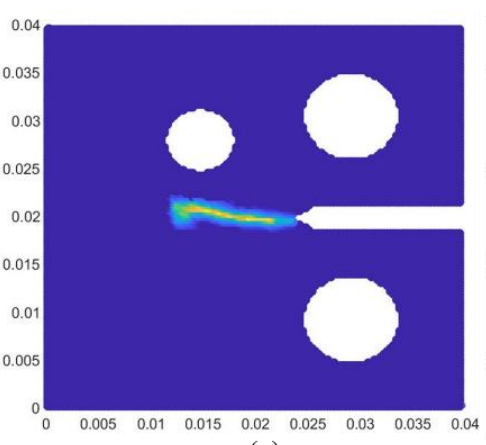

(a)

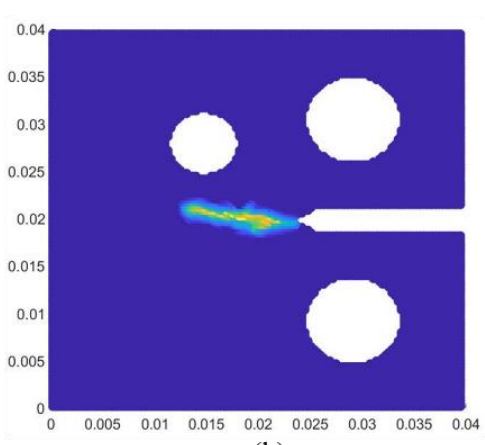

(b)

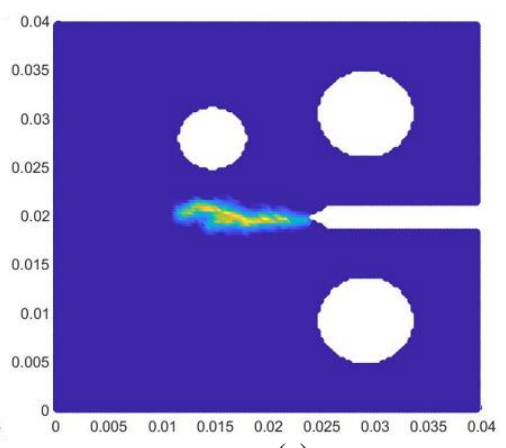

(c)

Fig. 16 Crack pattern of polycrystal for $\beta=2.0$. (a) 25 grains, $N=431 k$ cycles, (b) 100 grains, $N=453 k$ cycles, and (c) 400 grains, $N=512 k$ cycles .

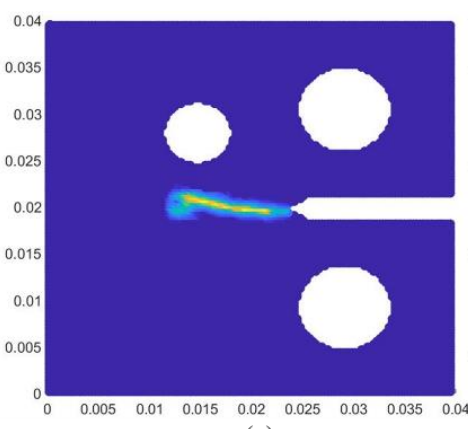

(a)

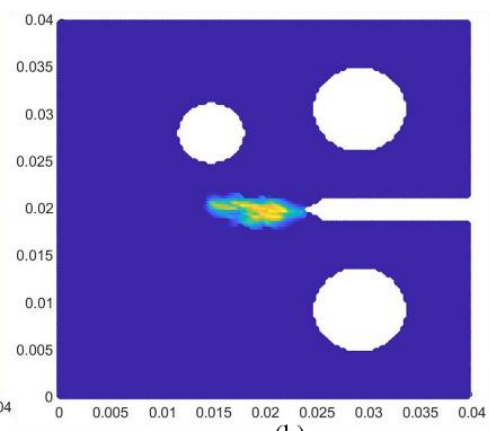

(b)

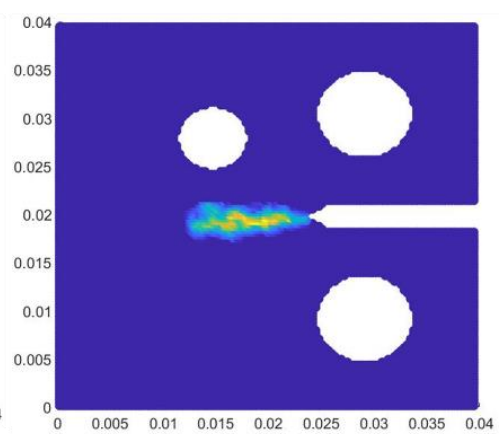

(c)

Fig. 17 Crack pattern of polycrystal when $\beta=10.0$. (a) 25 grains, $N=429 k$ cycles, (b) 100 grains, $N=784 k$ cycles, and (c) 400 grains, $N=846 k$ cycles . 


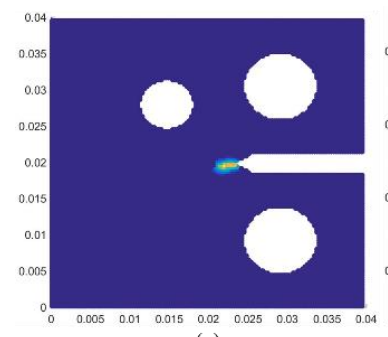

(a)

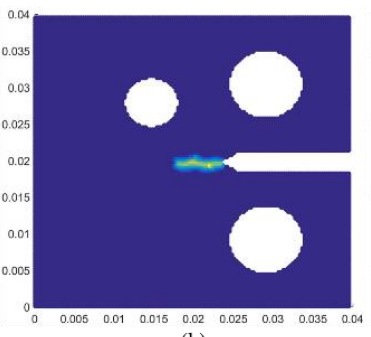

(b)

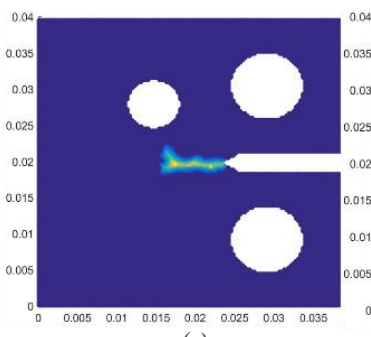

(c)

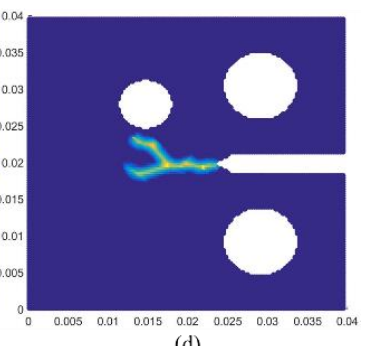

(d)

Fig. 18 Crack pattern of polycrystal when $\beta=0.1,100$ crystals, (a) $N=7.6 k$ cycles, (b) $N=22.5 k$ cycles, (c) $N=31.2 k$ cycles, and (d) $N=47.8 k$ cycles .

It is clearly seen in Fig. 18 that when $\beta=0.1$ the fracture in the polycrystal model is mainly inter-granular fracture and the crack propagates following the grain boundaries. However, when the crack tip reaches a certain key point as shown by red arrow in Fig. 10(b), the crack branches. This crack branching was also observed in the experimental results by Vasudevan et. al. (1984), Gilbert et. al. (1999) and Rios et. al. (1984).

\section{Conclusions}

In this study, fatigue analysis of polycrystalline materials was performed by using Peridynamic Theory with a novel crack tip detection algorithm. The effect of the interface strength coefficient on crack pattern and the effect of crystal size on crack propagation speed and crack branching are investigated. The new crack tip detection algorithm was validated by comparing against another study in the literature. The observations based on evaluated numerical results can be summarised as:

1. The change in the interface strength coefficient yields different fracture modes in the form of inter-granular and trans-granular fracture.

2. The size of crystals has a larger effect on the crack propagation speed for intergranular fractures than trans-granular fractures.

3. The crack branching in fatigue analysis can be automatically captured by the PD fatigue model for weaker grain boundary cases.

As a future study, the crack detection algorithm can be extended for a 3D model along with the PD fatigue model since the numerical studies in this paper mainly focused on 2D simulations.

\section{References}

Alpay, S. and Madenci, E., 2013. Crack growth prediction in fully-coupled thermal and deformation fields using peridynamic theory. In 54th AIAA/ASME/ASCE/AHS/ASC structures, structural dynamics, and materials conference (p. 1477). 
Askari, E, F Bobaru, RB Lehoucq, ML Parks, SA Silling, and O Weckner. 2008. "Peridynamics for multiscale materials modeling." Journal of Physics: Conference Series, 125(1), p. 012078.

Basoglu, M.F., Zerin, Z., Kefal, A. and Oterkus, E., 2019. A computational model of peridynamic theory for deflecting behavior of crack propagation with micro-cracks. Computational Materials Science, 162, pp.33-46.

Bouvard, Jean-Luc, Jean Louis Chaboche, F. Feyel, and F. Gallerneau. 2009. "A cohesive zone model for fatigue and creep-fatigue crack growth in single crystal superalloys." International Journal of Fatigue 31 (5):868-879.

Cervera, M., and M. Chiumenti. 2006. "Mesh objective tensile cracking via a local continuum damage model and a crack tracking technique." Computer methods in applied mechanics engineering 196 (1-3):304-320.

Cervera, Miguel, Luca Pelà, Roberto Clemente, and Pere Roca. 2010. "A cracktracking technique for localized damage in quasi-brittle materials." Engineering Fracture Mechanics 77 (13):2431-2450.

Chevalier, Jerome, Christian Olagnon, and Gilbert Fantozzi. 1999. "Subcritical crack propagation in 3Y-TZP ceramics: static and cyclic fatigue." Journal of the American Ceramic Society 82 (11):3129-3138.

Chowdhury, S.R., Roy, P., Roy, D. and Reddy, J.N., 2019. A modified peridynamics correspondence principle: Removal of zero-energy deformation and other implications. Computer Methods in Applied Mechanics and Engineering, 346, pp.530-549.

Dauskarat, Reinhold H., David B. Marshall, and Robert O. Ritchie. 1990. "Cyclic fatigue-crack propagation in magnesia-partially-stabilized zirconia ceramics." Journal of the American Ceramic Society 73 (4):893-903.

Dauskardt, R. H., W. Yu, and R. O. Ritchie. 1987. "Fatigue crack propagation in transformation-toughened zirconia ceramic." Journal of the American Ceramic Society 70 (10):C-248-C-252.

De Meo, Dennj, Ning Zhu, and Erkan Oterkus. 2016. "Peridynamic modeling of granular fracture in polycrystalline materials." Journal of Engineering Materials Technology 138 (4):041008.

Dhondt, Guido. 1998. "Automatic 3-D mode I crack propagation calculations with finite elements." International Journal for Numerical Methods in Engineering 41 (4):739-757.

Diyaroglu, C., Oterkus, E. and Oterkus, S., 2019. An Euler-Bernoulli beam formulation in an ordinary state-based peridynamic framework. Mathematics and Mechanics of Solids, 24(2), pp.361-376.

Donahue, Raymond J., H. McI. Clark, Phillip Atanmo, Raghvir Kumble, and Arthur J. McEvily. 1972. "Crack opening displacement and the rate of fatigue crack growth." International Journal of Fracture Mechanics 8 (2):209-219. 
Dong, P. . 2001. "A structural stress definition and numerical implementation for fatigue analysis of welded joints." International Journal of Fatigue 23 (10):865-876.

Dowling, NE, and JA Begley. 1976. "Fatigue crack growth during gross plasticity and the J-integral." In Mechanics of crack growth. ASTM International.

Fang, G., Liu, S., Fu, M., Wang, B., Wu, Z. and Liang, J., 2019. A method to couple state-based peridynamics and finite element method for crack propagation problem. Mechanics Research Communications, 95, pp.89-95.

Gerstle, Walter H., Luiz F. Martha, and Anthony R. Ingraffea. 1987. "Finite and boundary element modeling of crack propagation in two and three dimensions." Engineering with Computers 2 (3):167-183.

Gilbert, C. J., V. Schroeder, and R. O. Ritchie. 1999. "Mechanisms for fracture and fatigue-crack propagation in a bulk metallic glass." Metallurgical and Materials Transactions A 30 (7):1739-1753.

Gu, X., Zhang, Q., Madenci, E. and Xia, X., 2019. Possible causes of numerical oscillations in non-ordinary state-based peridynamics and a bond-associated higherorder stabilized model. Computer Methods in Applied Mechanics and Engineering, 357, p.112592.

Hopper, C. D., and K. J. Miller. 1977. "Fatigue crack propagation in biaxial stress fields." The Journal of Strain Analysis for Engineering Design 12 (1):23-28.

Hu, Y. L., and E. Madenci. 2017. "Peridynamics for fatigue life and residual strength prediction of composite laminates." Composite Structures 160:169-184.

Huang, X., Bie, Z., Wang, L., Jin, Y., Liu, X., Su, G. and He, X., 2019. Finite element method of bond-based peridynamics and its ABAQUS implementation. Engineering Fracture Mechanics, 206, pp.408-426.

Huffman, P. J., J. Ferreira, J. A. F. O. Correia, Abilio M. P. De Jesus, G. Lesiuk, Filippo Berto, A. Fernandez-Canteli, and G. Glinka. 2017. "Fatigue crack propagation prediction of a pressure vessel mild steel based on a strain energy density model." Frattura ed Integrita Strutturale 42:74-84.

Imachi, M., Tanaka, S., Ozdemir, M., Bui, T.Q., Oterkus, S. and Oterkus, E., 2020. Dynamic crack arrest analysis by ordinary state-based peridynamics. International Journal of Fracture, 221(2), pp.155-169.

Irwin, George R. 1957. "Analysis of stresses and strains near the end of a crack traversing a plate." J. appl. Mech. 24:351-369.

Kirane, Kedar, and Somnath Ghosh. 2008. "A cold dwell fatigue crack nucleation criterion for polycrystalline Ti-6242 using grain-level crystal plasticity FE model." International Journal of Fatigue 30 (12):2127-2139. 
Lindley, T. C., and C. E. Richards. 1974. "The relevance of crack closure to fatigue crack propagation." Materials Science Engineering 14 (3):281-293.

Liu, L., N. S. Husseini, C. J. Torbet, W-K Lee, R. Clarke, J. W. Jones, and T. M. Pollock. 2011. "In situ synchrotron X-ray imaging of high-cycle fatigue crack propagation in single-crystal nickel-base alloys." Acta Materialia 59 (13):5103-5115.

Liu, Z., Bie, Y., Cui, Z. and Cui, X., 2020. Ordinary state-based peridynamics for nonlinear hardening plastic materials' deformation and its fracture process.

Engineering Fracture Mechanics, 223, p.106782.

Madenci, Erdogan, and Erkan Oterkus. 2014. Peridynamic Theory and Its Applications. Vol. 17. New York: Springer.

Marines, I., G. Dominguez, G. Baudry, J.-F. Vittori, S. Rathery, J.-P. Doucet, and C. Bathias. 2003. "Ultrasonic fatigue tests on bearing steel AISI-SAE 52100 at frequency of 20 and $30 \mathrm{kHz}$." International Journal of Fatigue 25 (9-11):1037-1046.

Newman, J. C. 1981. "A crack-closure model for predicting fatigue crack growth under aircraft spectrum loading." In Methods and models for predicting fatigue crack growth under random loading. ASTM International.

Newman, James C. 1976. "A finite-element analysis of fatigue crack closure." In Mechanics of crack growth. ASTM International.

Nguyen, O., E. A. Repetto, Michael Ortiz, and R. A. Radovitzky. 2001. "A cohesive model of fatigue crack growth." International Journal of Fracture 110 (4):351-369.

Ni, T., Zaccariotto, M., Zhu, Q.Z. and Galvanetto, U., 2019. Static solution of crack propagation problems in Peridynamics. Computer Methods in Applied Mechanics and Engineering, 346, pp.126-151.

Oterkus, Erkan, Ibrahim Guven, and Erdogan Madenci. 2010. "Fatigue failure model with peridynamic theory." 2010 12th IEEE Intersociety Conference on Thermal and Thermomechanical Phenomena in Electronic Systems.

Oterkus, E., Guven, I. and Madenci, E., 2012. Impact damage assessment by using peridynamic theory. Open Engineering, 2(4), pp.523-531.

Paris, Paul C. 1961. "A rational analytic theory of fatigue." The trend in engineering 13:9.

Pearson, S. 1975. "Initiation of fatigue cracks in commercial aluminium alloys and the subsequent propagation of very short cracks." Engineering Fracture Mechanics 7 (2):235-247.

Potirniche, G. P., M. F. Horstemeyer, B. Jelinek, and G. J. Wagner. 2005. "Fatigue damage in nickel and copper single crystals at nanoscale." International Journal of Fatigue 27 (10-12):1179-1185. 
Proudhon, Henry, Jia Li, F. Wang, A. Roos, Vincent Chiaruttini, and Samuel Forest. 2016. "3D simulation of short fatigue crack propagation by finite element crystal plasticity and remeshing." International Journal of Fatigue 82:238-246.

Reece, Michael J., Frank Guiu, and Mohammad F. R. Sammur. 1989. "Cyclic fatigue crack propagation in alumina under direct tension - compression loading." Journal of the American Ceramic Society 72 (2):348-352.

Rice, J. 1967. "Mechanics of crack tip deformation and extension by fatigue." In Fatigue crack propagation. ASTM International.

Rios, E. R. DE LOS, Z. Tang, and K. J. Miller. 1984. "Short crack fatigue behaviour in a medium carbon steel." Fatigue \& Fracture of Engineering Materials \& Structures 7 (2):97-108.

Roe, K. L., and T. Siegmund. 2003. "An irreversible cohesive zone model for interface fatigue crack growth simulation." Engineering fracture mechanics 70 (2):209-232.

Saloustros, Savvas, Luca Pelà, and Miguel Cervera. 2015. "A crack-tracking technique for localized cohesive-frictional damage." Engineering Fracture Mechanics 150:96-114.

Sancho, José M., Jaime Planas, David A. Cendón, E. Reyes, and J. C. Gálvez. 2007. "An embedded crack model for finite element analysis of concrete fracture." Engineering Fracture Mechanics 74 (1-2):75-86.

Seleson, Pablo, and Michael Parks. 2011. "On the role of the influence function in the peridynamic theory." International Journal of Multiscale Computational Engineering 9 (6):689-706.

Shiozawa, K., L. Lu, and S. Ishihara. 2001. "S-N curve characteristics and subsurface crack initiation behaviour in ultra-long life fatigue of a high carbon-chromium bearing steel." Fatigue Fracture of Engineering Materials Structures 24 (12):781-790.

Sih, G. C., and B. M. Barthelemy. 1980. "Mixed mode fatigue crack growth predictions." Engineering Fracture Mechanics 13 (3):439-451.

Silling, Stewart A. 2000. "Reformulation of elasticity theory for discontinuities and long-range forces." Journal of the Mechanics and Physics of Solids 48 (1):175-209.

Silling, Stewart A, and Ebrahim Askari. 2005. "A meshfree method based on the peridynamic model of solid mechanics." Computers \& structures 83 (17-18):15261535 .

Silling, Stewart A., and Abe Askari. 2014. Peridynamic model for fatigue cracking. SAND2014-18590, Albuquerque, New Mexico 87185 and Livermore, California 94550: Sandia National Laboratories.

Sukumar, N., David L. Chopp, and B. Moran. 2003. "Extended finite element method and fast marching method for three-dimensional fatigue crack propagation."

Engineering Fracture Mechanics 70 (1):29-48. 
Tang, Tian, Sungho Kim, and M. F. Horstemeyer. 2010. "Fatigue crack growth in magnesium single crystals under cyclic loading: molecular dynamics simulation." Computational Materials Science 48 (2):426-439.

Vasudevan, A. K., P. E. Bretz, A. C. Miller, and S. Suresh. 1984. "Fatigue crack growth behavior of aluminum alloy 2020 (Al-Cu-Li-Mn-Cd)." Materials Science and Engineering 64 (1):113-122.

Von Euw, E. F. J., R. W. Hertzberg, and Richard Roberts. 1972. "Delay effects in fatigue crack propagation." Stress Analysis and Growth of Cracks: Proceedings of the 1971 National Symposium on Fracture Mechanics: Part 1.

Wang, Chen, and Xiwu Xu. 2015. "Cohesive element analysis of fatigue delamination propagation in composite materials with improved crack tip tracking algorism."

Composite Structures 134:176-184.

Wang, H., Oterkus, E. and Oterkus, S., 2018. Predicting fracture evolution during lithiation process using peridynamics. Engineering Fracture Mechanics, 192, pp.176191.

Wolf, Elber. 1970. "Fatigue crack closure under cyclic tension." Engineering Fracture Mechanics 2 (1):37-45.

Yang, B., S. Mall, and Krishnaswa Ravi-Chandar. 2001. "A cohesive zone model for fatigue crack growth in quasibrittle materials." International Journal of solids Structures 38 (22-23):3927-3944.

Yang, Z., Oterkus, E., Nguyen, C.T. and Oterkus, S., 2019. Implementation of peridynamic beam and plate formulations in finite element framework. Continuum Mechanics and Thermodynamics, 31(1), pp.301-315.

Zhai, Tong-guang, A. J. Wilkinson, and J. W. Martin. 2000. "A crystallographic mechanism for fatigue crack propagation through grain boundaries." Acta materialia 48 (20):4917-4927.

Zhang, Guanfeng, Quang Le, Adrian Loghin, Arun Subramaniyan, and Florin Bobaru. 2016. "Validation of a peridynamic model for fatigue cracking." Engineering Fracture Mechanics 162:76-94.

Zhang, Y., Deng, J., Deng, H. and Ke, B., 2019. Peridynamics simulation of rock fracturing under liquid carbon dioxide blasting. International Journal of Damage Mechanics, 28(7), pp.1038-1052.

Zheng, Xiulin, and Manfred A. Hirt. 1983. "Fatigue crack propagation in steels." Engineering Fracture Mechanics 18 (5):965-973.

Zhu, Ning, Dennj De Meo, and Erkan Oterkus. 2016. "Modelling of granular fracture in polycrystalline materials using ordinary state-based peridynamics." Materials 9 (12):977. 